HEAD AND NECK

\title{
Impact of a dedicated radiologist as a member of the head and neck tumour board: a single-institution experience
}

\author{
Impatto della presenza di un radiologo dedicato nella gestione multidisciplinare \\ dei pazienti con tumori del distretto testa collo: l'esperienza di un singolo centro
}

\begin{abstract}
Daniela Alterio', Lorenzo Preda ${ }^{2,3}$, Stefania Volpe ${ }^{1,4}$, Caterina Giannitto ${ }^{5}$, Giulia Riva ${ }^{1,4}$, Frank Arthur Kamga Pounou1,4, Murat Atac ${ }^{6}$, Gioacchino Giugliano ${ }^{6}$, Roberto Bruschini', Annamaria Ferrari', Giulia Marvaso ${ }^{1}$, Maria Cossu Rocca ${ }^{7}$, Elena Verri ${ }^{7}$, Duccio Rossi ${ }^{8}$, Massimo Bellomi ${ }^{4,5}$, Barbara Alicja Jereczek-Fossa ${ }^{1,4}$, Roberto Orecchia ${ }^{9}$, Mohssen Ansarin ${ }^{6}$

' Department of Radiation Oncology, IEO, European Institute of Oncology IRCCS, Milan, Italy; ${ }^{2}$ Department of Clinical-Surgical, Diagnostic and Pediatric Sciences, University of Pavia, Italy; ${ }^{3}$ Diagnostic Imaging Unit, National Center of Oncological Hadron Therapy (CNAO), Pavia, Italy; ${ }^{4}$ Department of Oncology and Hemato-Oncology, University of Milan, Italy; ${ }^{5}$ Department of Radiology, IEO, European Institute of Oncology IRCCS, Milan, Italy; ${ }^{6}$ Department of Head and Neck Surgery, IEO, European Institute of Oncology IRCCS, Milan, Italy; ${ }^{7}$ Department of Head and Neck and Urogenital Medical Oncology, IEO, European Institute of Oncology IRCCS, Milan, Italy; ${ }^{8}$ Postgraduate School of Radiology, University of Milan, Italy; ${ }^{9}$ Scientific Directorate, IEO, European Institute of Oncology IRCCS, Milan, Italy
\end{abstract}

\section{SUMMARY}

The aim of this study was to quantify the impact of radiologic image review performed by experienced radiologists in a multidisciplinary team (MDT) for head and neck cancers (HNCs). We performed a retrospective review of cases discussed at MDT meetings from April 2014 to March 2017 for which radiologic review was required. All changes in the former radiologic report were collected and classified as follows: 1) modifications of radiological reports (patients for whom the treatment strategy had not been defined at the moment of MDT meeting) and 2) modifications in treatment strategy (patients for whom treatment strategy had previously been defined and subsequently modified according to the outcome of radiologic revision). The latter subgroup was further categorised as "major changes" and as "minor changes". A total of 540 cases were retrieved. Imaging review was required at the time of tumour diagnosis in $310(57.4 \%)$ cases. Most patients $(69 \%)$ had advanced stage tumours (III and IV). In 262 (48\%) cases, no change of the initial radiologic report was made. In a total of $144(27 \%)$ cases, the available imaging was not considered sufficient for a final indication to treatment and further imaging was required. In the remaining $134(25 \%)$ cases, radiologic review led to a modification of either tumour staging $(55 \%)$ or treatment strategy (45\%). Specifically, major and minor modifications were applied in $44(13 \%)$ and $17(11 \%)$ of the cases considered, respectively. Among 134 patients for whom the radiologic review led to stage/treatment modification, follow-up was available for 118. In all but one patient, we could confirm the original reports were correctly modified per MDT discussion results. Our data strongly support the importance of including an experienced radiologist as a core member of the MDT for HNCs.

KEY WORDS: head and neck cancer, multidisciplinary management, imaging, radiology

\section{RIASSUNTO}

Scopo del lavoro è stato quello di quantificare l'impatto della revisione radiologica condotta da radiologi esperti nell'ambito di un gruppo di lavoro multidisciplinare nella gestione dei tumori del distretto testa collo. È stata condotta un'analisi retrospettiva dei casi discussi nelle riunioni multidisciplinari svoltesi tra i mesi di aprile 2014 e marzo 2017, per i quali è stata richiesta una revisione radiologica. Le modifiche apportate ai referti radiologici originari sono state classificate come segue: 1) variazioni dello stadio tumorale e 2) cambiamenti alla strategia terapeutica, di seguito classificati come "rilevanti" e come "non rilevanti". La revisione radiologica si è resa necessaria in 540 casi, in 310 (57,4\%) si trattava di pazienti in prima diagnosi. La maggior parte dei pazienti (69\%) presentava
Received: September 10, 2019

Accepted: September 27, 2019

Correspondence

Lorenzo Preda

Fondazione CNAO, strada Campeggi 53, 27100

Pavia, Italy

Tel. +390382 078613. Fax +390382078901

E-mail: lorenzo.preda@cnao.it

Funding

None.

Conflict of interest

The Authors declare no conflict of interest.

How to cite this article: Alterio D, Preda L, Volpe S, et al. Impact of a dedicated radiologist as a member of the head and neck tumour board: a single-institution experience. Acta Otorhinolaryngol Ital 2020;40:26-32. https:// doi.org/10.14639/0392-100X-N0326

(C) Società Italiana di Otorinolaringoiatria e Chirurgia Cervico-Facciale

\section{cc) (i) $(9)$}

This is an open access article distributed in accordance with the CC-BY-NC-ND (Creative Commons Attribution-NonCommercial-NoDerivatives 4.0 International) license. The article can be used by giving appropriate credit and mentioning the license, but only for non-commercial purposes and only in the original version. For further information: https:// creativecommons.org/licenses/by-nc-nd/4.0/deed.en 
tumori in stadio avanzato (III e IV). In 262 casi (48\%) non è stato apportato alcun cambiamento rispetto alla valutazione radiologica iniziale. In 144 casi (27\%), le immagini disponibili non sono state considerate sufficienti per una adeguata pianificazione terapeutica e sono state richieste ulteriori indagini diagnostiche. Nei rimanenti 134 casi (25\%), la revisione radiologica ha portato a modificare lo stadio della malattia $(55 \%)$ o la strategia terapeutica (44\%). In particolare, variazioni "rilevanti" $e$ "non rilevanti" alla strategia terapeutica sono state applicate rispettivamente in $44(13 \%)$ e in $17(11 \%)$ dei casi considerati. In 118 di tali pazienti erano disponibili i successivi controlli nel tempo. In tutti $i$ casi, tranne uno, è stato possibile confermare che la modifica apportata ai referti originari in sede di discussione multidisciplinare era corretta. I nostri dati confermano l'importanza di includere un radiologo esperto come componente fondamentale dei gruppi di lavoro multidisciplinari per la gestione dei tumori del distretto testa-collo.

PAROLE CHIAVI: neoplasie laringologiche, gestione multidisciplinare, diagnostica per immagini, radiologia

\section{Introduction}

Head and neck cancers (HNCs) are a heterogeneous group of malignancies, which may arise in multiple subsites of an anatomically complex region. Despite the fact that national and international guidelines have led to a progressive standardisation of indications for treatment, the availability of different equipment and expertise (e.g. transoral robotic surgery) may vary between single institutions, thus influencing treatment strategies. Moreover, as multimodality treatments have become the standard of care in locally advanced-stage disease, integrated management between different medical specialties has become paramount ${ }^{1,2}$.

Multidisciplinary care has been shown to bring several advantages to patient management, including but not limited to shorter diagnosis-treatment time, greater adherence to clinical guidelines and optimisation of diagnostic and treatment procedures ${ }^{3-5}$. Arguably, the combined effect of these factors may account for the better oncological outcomes of patients managed by multidisciplinary teams (MDTs) compared to those treated by a single referring physician ${ }^{6-9}$. These data have encouraged the incorporation of multidisciplinary management into national and international guidelines on treatment of $\mathrm{HNCs}{ }^{10,11}$.

In agreement with this statement, in 2000 our institution established an ongoing MDT program to coordinate the different phases of $\mathrm{HN}$ patient management ranging from diagnosis to supportive care. MDT meetings are arranged according to a weekly schedule and involve radiation oncologists, radiologists, ear-nose-throat (ENT) surgeons and medical oncologists with well-recognised clinical and scientific expertise in the field. Additional professionals have gradually entered the core of the MDT, including pathologists, dental surgeons, endocrinologists, speech therapists and dieticians. Due to the increasing availability of both morphological and functional imaging in HN patient workup ${ }^{12-14}$, the role of the radiologist has become paramount in every step of the clinical pathway.

Starting from these observations, the aim of the present study is to quantify the impact of inserting a radiologic image review into the pattern of care of HNC patients and to verify whether such an imaging-based indication was adequately tailored based on the analysis of subsequent follow-up.

\section{Materials and methods}

At our institution, radiologists with specific expertise in HNC have become steady core members of the MDT starting from 2010. Routinely, imaging may be reviewed both in the diagnostic setting and follow-up period for patients eligible either for surgical or a non-surgical approach. In the diagnostic setting, imaging reviews are performed to: 1) discriminate between benign and malignant lesions, 2) to refine tumour staging, and 3) to define the optimal treatment strategy, especially where radiological and clinical findings conflict. Conversely, if imaging review is required during the course of follow-up, the radiologist may be required to address at least one diagnostic issue from among the following: 1) disease persistence after a non-surgical strategy; 2) loco-regional recurrence and/or distant metastatic progression; 3) second primary tumour of the $\mathrm{HN}$ region; 4) surgical and/or radiotherapy sequelae (including osteoradionecrosis). Imaging review may be required either for examinations performed at our institution (if evaluated by a non-dedicated $\mathrm{HN}$ radiologist) or as a referral/second opinion for patients treated in other centres (with radiologic images evaluated by general radiologists). In those latter cases, reports from outside hospitals were available in the majority of cases. Whenever radiologic image review is required, both clinical information and DICOM (Digital Imaging and COmmunications in Medicine) files (CT and/ or MR) are provided to the radiologist at least one day before the MDT discussion for primary assessment. For the aim of the current analysis, we retrospectively reviewed all cases discussed at MDT meetings from April 2014 to March 2017. Of those, we selected only cases for whom a radiologic review of $\mathrm{CT}$ and/or MR was performed by two experienced radiologists.

The radiologists involved in the image review process were LP and CG. Although there are no precise criteria to define radiologist "expertise" on evaluating radiologic images of 
head and neck cancers, different parameters are generally considered: years of work on the topic, number of radiologic images examined, availability of modern radiologic equipment, the expertise of the centre (high volume centres), availability of certification endorsed by scientific societies and involvement in multidisciplinary discussions. LP has 15 years'experience in executing and reporting $\mathrm{CT}$ and MR HN examinations. Moreover, in 2013 he acquired the subspecialisation in Head and Neck Radiology endorsed by the European Society of Radiology. This qualification attests a standard of in-depth knowledge in the field of head and neck radiology. CG has 5 years' experience in executing and reporting CT and MR HN examinations. During the period considered, their referral Department was the Department of Diagnostic Imaging of the European Institute of Oncology which performs about $2600 \mathrm{HN}$ radiologic exams per year including approximately 220 MRs per year, 150 CTs per year and 2300 ultrasounds per year. LP and CG followed about $80 \%$ of the HN TC and MR exams of the Department. Both radiologists were fully involved in the MDT discussion of all cases requiring radiologic images revision. The study investigators were independent of the MDT radiologists.

Inclusion criteria were as follows: 1) clinical and/or radiological suspicion or histologically-proven diagnosis of HNC; 2) availability of a written medical record of the MDT discussion and final radiological report; 3) follow-up length of at least 12 months; 4) willingness and ability to sign a written informed consent for the use of personal data for educational and scientific purposes. Both malignant and benign tumour cases were included. Only CT and MR imaging were considered; data on positron emission tomography (PET) were also collected. Patients who were discussed regarding adjuvant treatment (i.e. radiation treatment alone or in association with systemic treatment) and/ or diagnosis of thyroid cancers were excluded.

The aim of the study was to quantify the impact of radiologic image review performed during MDT weekly discussion. Therefore, for each case, the original radiological report was compared to the result from the imaging reviewing process. The following descriptors were considered: 1) any changes in radiological reports in patients for whom treatment strategy had not been previously defined (Tumour Node Metastasis (TNM) staging system $7^{\text {th }}$ ed. in cases of staging modification); 2) any changes in treatment strategy. The latter were categorised as either "major modifications" (i.e. MDT non-indication to surgery in favour of radiotherapy \pm systemic therapy, and vice versa) or "minor modifications" (i.e. MDT indication to an alternative approach: open versus than minimally-invasive surgery, and

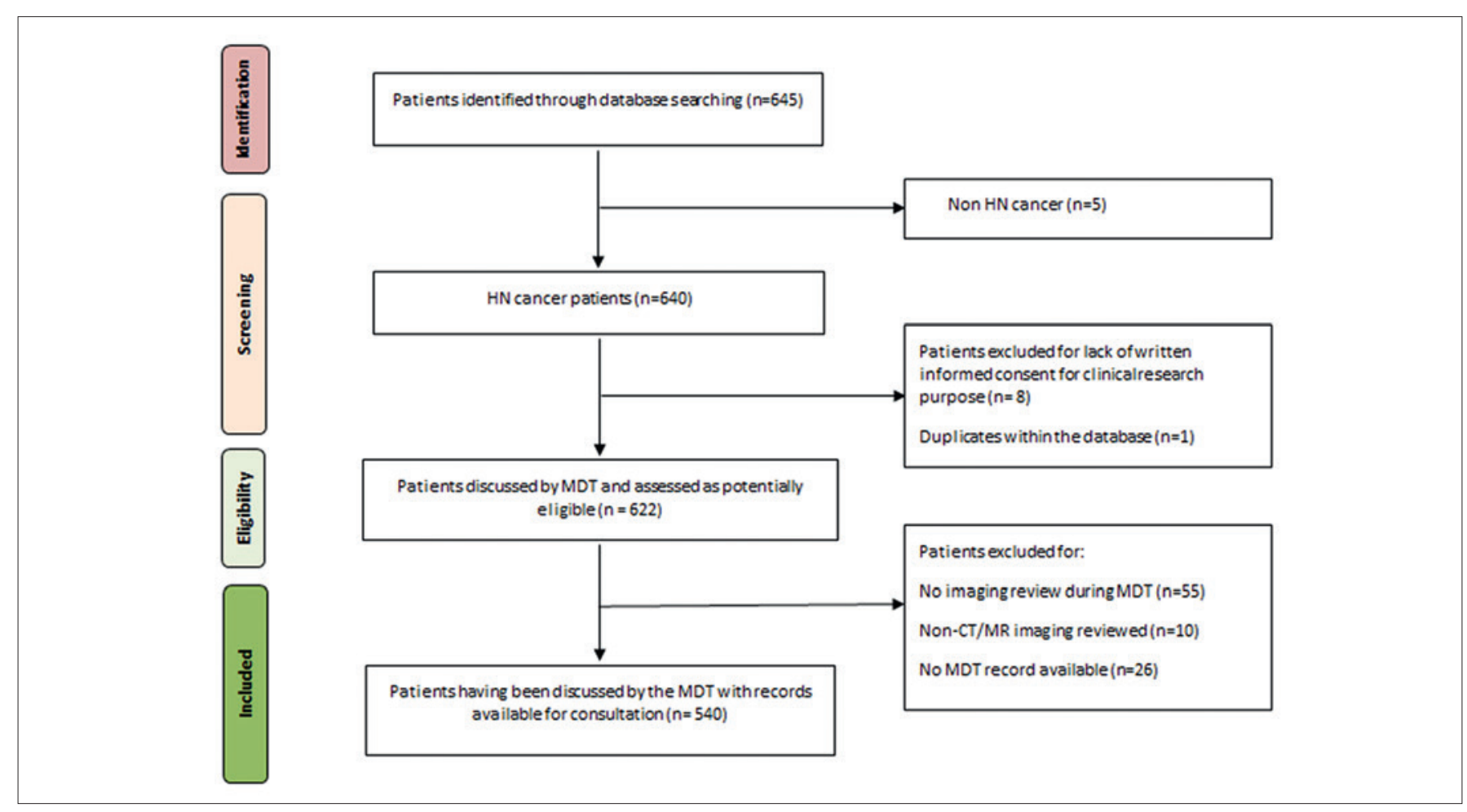

Figure 1. Consolidated Standards for Reporting Trials (CONSORT) diagram. 
vice versa). Additionally, MDT final decision and accuracy of MDT indication-to-treatment (as evaluated by histological confirmation and/or follow-up data) were collected.

Patient- and disease-related characteristics were retrieved from electronic medical records. DICOM files were retrospectively reviewed for verification of complex cases (LP, CG).

Descriptive statistical analysis was performed with the SAS statistical software (version 9.2; SAS Institute Inc, Cary, NC).

\section{Results}

A total of 2445 cases were discussed during the institutional MDT meetings over the period considered. Radiologic imaging review was required in 649 (26\%) cases; of those, 540 met inclusion criteria for the current analysis and constituted the final patient cohort. Details of case selection are provided by the CONSORT diagram depicted in Figure 1. Patients were predominantly male (66\%), median age was 63 (interquartile range, IQR: 52.9-71.6) years. The most represented HN subsite was the oropharynx (28\%), followed by the larynx (19\%) and oral cavity (18\%). Further patient demographics and disease-related details are provided in Table I.

Imaging review was required at the time of tumour diagnosis in 310 (57.4\%) cases. Most patients (69\%) had advanced stage (III and IV) tumours. The remaining 230 $(42.6 \%)$ patients were discussed during the course of follow-up, mainly because of suspected loco-regional recurrence (131 cases, 57\%). Among all cases discussed, 124 (23\%) represented the second opinion of patients referred from another centres. Specifics on the setting of imaging review are summarised in Table II.

Considering imaging modality, CT and MR were almost equally represented in our cohort, with $234(43 \%)$ and 249 $(46 \%)$ of cases, respectively, as illustrated in Table III. Patient stratification according to MDT decision following radiologic imaging review is summarised in Figure 2.

Details of the miscellaneous group (patients for whom radiologic revision did not lead to any amendment in treatment strategy) are as follows:

- Patients with prior indication to surgery for whom the TNM staging modification did not translate into a modification of the treatment strategy (56 patients 77\%). The accuracy of staging modification could be verified through the pathological report, which confirmed adequacy in all cases.

- Patients for whom the diagnostic question was to differentiate between benign and malignant lesions (11 patients, $14 \%)$. Of these, 6 patients (59\%) underwent fur-
Table I. Patient and disease characteristics.

$\begin{array}{lc}\text { Total number of patients } & \begin{array}{c}\text { Number of patients } \\ \text { (\%) }\end{array} \\ \text { Gender } & 540 \\ \text { M } & \\ \text { F } & 358(66) \\ \end{array}$

Age (at the time of multidisciplinary discussion)

Mean (range) year

$61.8(20-90)$

Median (IQR) year

$63.0(52.9-71.6)$

\section{Head and Neck sites}

\begin{tabular}{lc} 
Oral cavity & $98(18)$ \\
Oropharynx & $152(28)$ \\
Hypopharynx & $24(4)$ \\
Larynx & $100(19)$ \\
Salivary glands & $60(11)$ \\
Skin & $17(3)$ \\
Nasal cavity and paranasal sinuses & $16(3)$ \\
Lymph node metastasis from unknown primary & $12(2)$ \\
Recurrences of nasopharyngeal tumours & $5(1)$ \\
Other head and neck sites & $56(11)$ \\
Histology & \\
Squamous cell carcinoma & $391(72)$ \\
Adenocarcinoma & $15(3)$ \\
Adenoid cystic carcinoma & $23(4)$ \\
Pleomorphic adenoma & $17(3)$ \\
Mucoepidermoid tumours & $6(1)$ \\
Sarcoma & $9(2)$ \\
Schwannoma & $2(0.5)$ \\
Paraganglioma & $2(0.5)$ \\
Neuroendocrine tumours & $3(0.6)$ \\
Benign histology & $9(2)$ \\
Other histology & $17(3)$ \\
N.A. at the time of discussion & $46(9)$ \\
\hline rang: cancer of the unknown primary; HNC: head and neck cancer; IQR: interquartile \\
\end{tabular}

ther assessment through fine needle aspiration or biopsy, while 3 patients (25\%) received an indication for clinical and/or radiological follow-up. For these 9 patients, modification of the radiologic report proved to be correct in all cases. As the remaining 2 patients (16\%) were lost to follow-up, it was not possible to verify the appropriateness of the radiologic report modification.

- Patients for whom the diagnostic question was to differentiate between recurrent/persistent disease and treatment-related sequelae (i.e. local recurrence vs post-RT osteoradionecrosis) (6 patients, 9\%). Pathological confirmation was required in 4 cases, and the modification 
Table II. Aim of the radiologic image revision, sorted by first-diagnosis and follow-up settings.

\begin{tabular}{|c|c|c|}
\hline Setting of radiologic revision & Number of patients (\%) & \\
\hline \multirow[t]{4}{*}{ Diagnosis } & & $310(57.4)$ \\
\hline & Malignant tumours: early-stage disease & $59(19)$ \\
\hline & Malignant tumours: advanced-stage disease & $216(70)$ \\
\hline & Benign tumours & $35(11)$ \\
\hline \multirow[t]{9}{*}{ Follow-up } & & $230(42.6)$ \\
\hline & Suspected persistent disease & $23(10)$ \\
\hline & Suspected loco-regional recurrence & $130(57)$ \\
\hline & Suspected distant metastasis & $19(8)$ \\
\hline & Suspected loco-regional recurrence and distant metastasis & $12(5)$ \\
\hline & Suspected second primary tumour & $28(12)$ \\
\hline & Suspected surgical or radiation-sequelae (including osteoradionecrosis) & $8(3)$ \\
\hline & Surgical- or radiation sequelae versus locoregional recurrence & $3(2)$ \\
\hline & Other & $7(3)$ \\
\hline
\end{tabular}

Table III. Type of images reviewed by the multidisciplinary team.

\begin{tabular}{lc} 
Imaging modality & No. of patients (\%) \\
CT & $234(43)$ \\
MR & $249(46)$ \\
CT+MR & $25(4.5)$ \\
CT+PET & $14(3)$ \\
MR+PET & $15(3)$ \\
CT+MR+PET & $3(0.5)$ \\
\hline CT: computed tomography: MR: magnetic resonance. PET: positron emission tomography
\end{tabular}

of the initial radiologic report was proven to be accurate in all but one case (the detailed description of this case is provided later in the section). Of the remaining 2 cases, modification of the radiologic report was confirmed by subsequent imaging in one case, while the other case could not be verified as the patient was lost to follow-up. Missing follow-up data represented 21\% (13/61) for patients for whom radiologic image revision led to a modification in treatment strategy.

Overall, considering the whole cohort of 540 patients, imaging review led to modification of radiologic reports (miscellaneous group) or in treatment strategy in $73(13 \%)$ and $61(11 \%)$ of cases, respectively.

Follow-up was available for 118 (86\%) of the 134 patients for whom the radiological report was edited following the MDT meeting. Forty-five $(37 \%)$ patients were submitted to subsequent surgery or cytohistologic sampling (specifically, 50\% underwent surgical specimen examination, $35 \%$ were biopsied and $15 \%$ received a fine-needle biopsy). For the remaining 73 patients, the adequacy of radiologic indication was verified through further imaging and/or clinical examination during the follow up period.

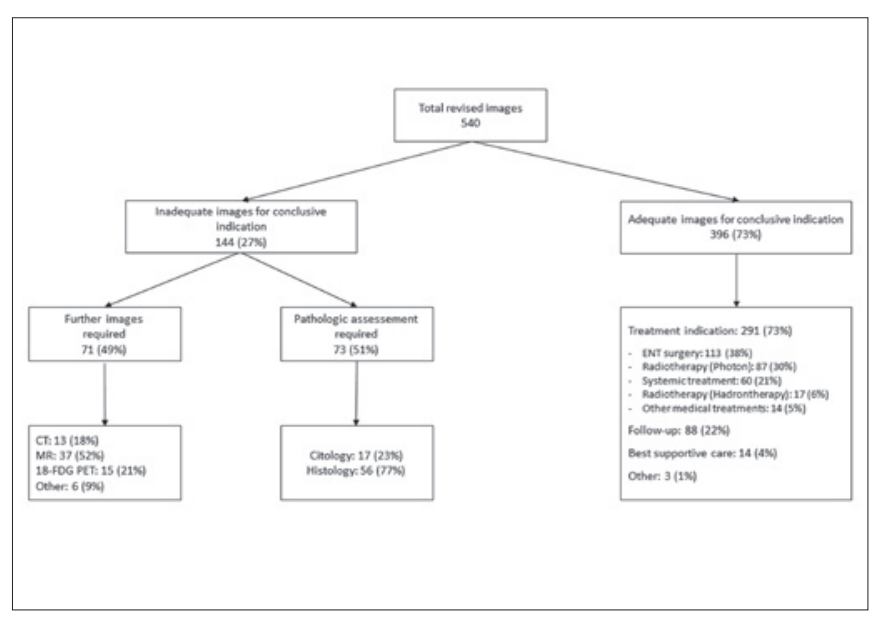

Figure 2. Schematic representation of cases stratification according to MDT decisions (tumour staging and indication-to-treatment).

Finally, among patients with available follow up (134 cases) in $117(87 \%)$ we could confirm that modification of the original reports as defined during the MDT discussion was correct.

In only one case was the modification of initial radiological chart not confirmed by subsequent histologic specimen examination. This was the case of a 70-year-old man with a diagnosis of early stage (cT2cNOM0) glottic cancer treated with curative radiotherapy followed by laser surgery for persistent disease. One year after the end of radiotherapy, a CT scan showed a partially necrotic tissue in the right residual larynx with transglottic extension. The finding was considered to be radiologically stable compared with two previous exams and was therefore considered as a radiation-related sequela. Nevertheless, because of worsening symptoms and clinical evidence of cricoid cartilage expo- 
sure, after a second MDT discussion, the patient was indicated for a total laryngectomy. The laryngectomy specimen revealed the presence of persistent malignancy.

The management of cases presented for radiological review is depicted in Figure 3. In particular, available imaging could be deemed inadequate and/or insufficient for appropriate evaluation either due to technical issues (such as low-quality image) or to the need for integrated morpho-functional information. In these cases, continuation of the diagnostic workup or cytohistologic assessment was required. Conversely, when the available data were considered adequate, patients were referred for either treatment, follow-up, or best supportive care according to the MDT decision.

A time-trend analysis documented an increase in the absolute number of cases presented to the MDT for radiologic consultation during the period under consideration (Tab. IV).

\section{Discussion}

The results of this retrospective analysis show that HN image review performed in a multidisciplinary context has a significant influence on clinical management as it gave rise to a modification of the initial radiologic report in one out of four cases, and led to a change in tumour treatment strategy in $11 \%$ of cases. Moreover, our work highlights the high quality of the image review provided, since the majority of the suggested modifications were confirmed by subsequent follow up and/or pathologic specimen examination.

The importance of radiologists at the core of the MDT has been progressively advocated ${ }^{3,5,15,16}$. Not only has the availability of radiologic imaging grown, but recent technological advances in imaging acquisition and post-processing elaboration also require an increasing level of expertise. In the era of precision medicine, the definition of tumour volume and extent is of paramount importance to define the best treatment approach and to avoid geographically missing the target lesion (i.e. mini-invasive surgery, intensity-modulated and stereotactic body radiation therapy). Moreover, the availability of radiologic imaging with higher sensitivity and specificity during follow-up could result in selecting patients whose loco-regional recurrences are being diagnosed at an early stage, thus making them potentially good candidates for curative-intent salvage treatments (surgery and/or re-irra-

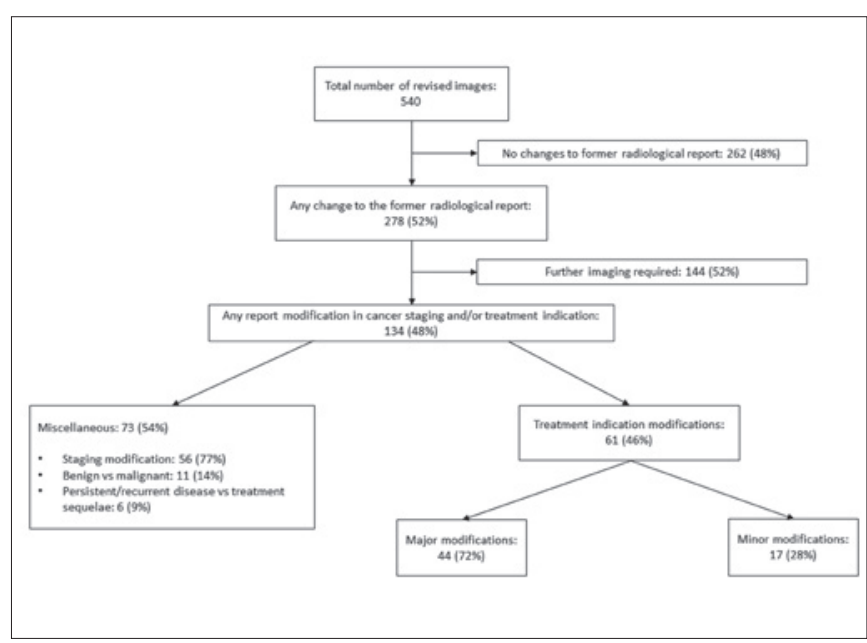

Figure 3. Schematic representation of MDT decision outline.

diation). Bergamini et al. ${ }^{3}$ found that MDT evaluation led to staging refinement or modifications of the original treatment plan in approximately $60 \%$ of HNC cases; of those, $49 \%$ were indications to further diagnostic imaging, pathology assessment and/or molecular analyses. Our results are in line with these observations. Accordingly, a recent systematic review of 27 studies by Pillay et al. ${ }^{6}$ has shown that the MDT discussion led to changes in former diagnostic reports in $4 \%$ to $45 \%$ of cases, with the significant limitation that only one of the included studies was dedicated to HNC. The results of our analysis are in line with those observations since accurate review of the radiologic images led to a modification of tumour staging and/or treatment strategy in $13 \%$ and $11 \%$ of patients, respectively.

Friedland et al. ${ }^{7}$ reported that patients seen by the MDT were more likely to present with advanced stage disease compared to those evaluated by individual specialists. Interestingly, the investigators demonstrated a statistically significant benefit for stage IV patients discussed by the MDT, as they were more frequently eligible for multimodality treatment and therefore could achieve better 5-year survival compared with non-MDT patients. This observation is confirmed by our series in which the majority of cases requiring a radiologic image review had advanced stage tumours (69\% stage III and IV), corroborating that

Table IV. Number of cases discussed with radiologic image revision compared to the total of patient discussed during the multidisciplinary team meetings, temporal trend/year.

\begin{tabular}{lcc} 
Considered period & $\begin{array}{c}\text { No. of patients } \\
\text { discussed }\end{array}$ & $\begin{array}{c}\text { Radiologic image revision } \\
\text { (\% of patients discussed) }\end{array}$ \\
\hline April 2014 - March 2015 & 735 & $113(15)$ \\
April 2015 - March 2016 & 804 & $195(24)$ \\
April 2016 - March 2017 & 906 & $21(23)$ \\
\hline
\end{tabular}


the multidisciplinary approach is mostly required in cases for which a multimodality treatment strategy is mandatory. Since we did not have a comparative group of patients managed by a single specialist, we were not able to quantify the impact of MDT management on patients' outcome.

A previously published report by Loevner et al. analysed issues similar to those analysed in the present paper for head and neck cancer patients. In this retrospective study, authors showed that in 56 of 136 cases (41\%) the original reports were modified after re-evaluation by an expert radiologist with a significant impact on subsequent patient management ${ }^{17}$. Of note, the study involved patients treated in the 2000s. Despite the significant technical advances in radiologic imaging in recent decades, the role of a dedicated radiologist in a MDT has been strongly confirmed by the present study including a larger cohort, since a comparable proportion of radiologic reports $(48 \%)$ have been changed after the image review given by an expert head and neck radiologist. All these data highlight the importance of having an expert radiologist present during multidisciplinary discussions, suggesting that radiologic images of every patient should be reviewed in order to optimise the treatment strategy.

\section{Conclusions}

We are well aware of the limitations of our study, including: 1) the retrospective nature of the analysis, 2) the exclusion of FDG-PET images from the radiologic revision in some patients, and 3) the unblinded evaluation of the initial radiologic report. Moreover, each MDT discussion also took into account other findings such as clinical examination, symptoms and clinical history. The final MDT decision was, therefore, a multidimensional process in which the radiologic images review represented only one, albeit fundamental, step. Nevertheless, we strongly believe our analysis may convey useful information not only towards the full integration of expert radiologists in the core of MDT for HNCs, but also towards a stronger standardisation of radiologic imaging acquisition and reporting. Although retrospective in nature, the strength of our work resides in the prospective collection of data related to all MDT decision. Moreover, as a tertiary care centre, follow-up information was available for the vast majority of cases, thus permitting the adequacy of the radiologic image review to be verified. In conclusion, in one of four cases in our cohort radiology review led to modification of staging/treatment strategy. This finding strongly supports the importance of including a dedicated HN radiologist as a core member of the MDT. Further efforts of prospective nature are warranted in order to assess whether imaging review in the setting of MDTs translates into improved oncological outcomes.

\section{References}

1 Bossi P, Alfieri S. The benefit of a multidisciplinary approach to the patient treated with (chemo) radiation for head and neck cancer. Curr Treat Options Oncol 2016;17:53. https://doi.org/10.1007/s11864016-0431-3

2 Kelly SL, Jackson JE, Hickey BE, et al. Multidisciplinary clinic care improves adherence to best practice in head and neck cancer. Am J Otolaryngol 2013;34:57-60. https://doi.org/10.1016/j.amjoto.2012.08.010

3 Bergamini C, Locati L, Bossi P, et al. Does a multidisciplinary team approach in a tertiary referral centre impact on the initial management of head and neck cancer? Oral Oncol 2016;54:54-7. https://doi. org/10.1016/j.oraloncology.2016.01.001

4 Brunner M, Gore SM, Read RL, et al. Head and neck multidisciplinary team meetings: effect on patient management. Head Neck 2015;37:1046-50. https://doi.org/10.1002/hed.23709

5 Stalfors J, Lundberg C, Westin T. Quality assessment of a multidisciplinary tumour meeting for patients with head and neck cancer. Acta Otolaryngol (Stockh) 2007;127:82-7. https://doi. org/10.1080/00016480600740589

6 Pillay B, Wootten AC, Crowe H, et al. The impact of multidisciplinary team meetings on patient assessment, management and outcomes in oncology settings: a systematic review of the literature. Cancer Treat Rev 2016;42:56-72. https://doi.org/10.1016/j.ctrv.2015.11.007

7 Friedland PL, Bozic B, Dewar J, et al. Impact of multidisciplinary team management in head and neck cancer patients. Br J Cancer 2011;104:1246-8. https://doi.org/10.1038/bjc.2011.92

8 Taylor C, Munro AJ, Glynne-Jones R, et al. Multidisciplinary team working in cancer: what is the evidence? BMJ 2010;340:c951-c951. https://doi.org/10.1136/bmj.c951

9 Andreano A, Ansarin M, Alterio D, et al. Indicatori di aderenza nel percorso di cura nei tumori del distretto cervico-facciale ottenuti da dati del registro tumori e flussi informativi sanitari. Acta Otorhinolaryngol Ital 2018;38:13-23. https://doi.org/10.14639/0392-100X-1934

10 Head and Neck Cancers. NCCN Clinical Practice Guidelines. Version 2.2018- June 20, 2018.

11 Associazione Italiana di Oncologia Medica, editor. Tumori della Testa e del Collo- Linee Guida 2016

12 Davis AJ, Rehmani R, Srinivasan A, et al. Perfusion and permeability imaging for head and neck cancer: theory, acquisition, postprocessing, and relevance to clinical imaging. Magn Reson Imaging Clin N Am 2018;26:19-35. https://doi.org/10.1016/j.mric.2017.08.002

13 Pérez-Lara A, Forghani R. Spectral computed tomography: technique and applications for head and neck cancer. Magn Reson Imaging Clin N Am 2018;26:1-17. https://doi.org/10.1016/j.mric.2017.08.001

14 Zhou Q, Zeng F, Ding Y, Fuller C, Wang J. Meta-analysis of diffusion-weighted imaging for predicting locoregional failure of chemoradiotherapy in patients with head and neck squamous cell carcinoma. Mol Clin Oncol 2018;8:197-203. https://doi.org/10.3892/mco.2017.1504

15 National Institute for Health and Care Excellence. Head and neck cancer - quality statement 4 : choice of treatment. 2017.

16 Chan ATC, Grégoire V, Lefebvre J-L, et al. Nasopharyngeal cancer: EHNS-ESMO-ESTRO Clinical Practice Guidelines for diagnosis, treatment and follow-up. Ann Oncol Off J Eur Soc Med Oncol 2012;23(Suppl 7):vii83-85. https://doi.org/10.1093/annonc/mds266

17 Loevner LA, Sonners AI, Schulman BJ, et al. Reinterpretation of cross-sectional images in patients with head and neck cancer in the setting of a multi-disciplinary cancer center. AJNR Am J Neuroradiol 2002;23:1622-6. 